Check for updates

Cite this: Phys. Chem. Chem. Phys., 2017, 19, 25961

Received 19th June 2017, Accepted 4th September 2017 DOI: $10.1039 / c 7 c p 04122 e$ rsc.li/pccp

\section{Coherent evolution of singlet spin states in PHOTO-PHIP and M2S experiments}

\begin{abstract}
A. N. Pravdivtsev, (D *ab A. V. Yurkovskaya, (D) ab P. A. Petrov (iD bc and H.-M. Vieth (D) ${ }^{\text {ad }}$
A consistent theoretical description of the spin dynamics underlying photo-PHIP (para-hydrogen induced polarization) experiments is given and validated experimentally: spectra from zero-quantum coherence (ZQC) "in-phase" and "out-of-phase" were obtained and evolution of ZQCs and the population of singlet spin state was tracked and modeled. Data from recent literature [O. Torres et al., J. Am. Chem. Soc., 2014] are reinterpreted. Advantages of using M2S sequences such as APSOC for detecting photo-PHIP are demonstrated. A sequence for 2D ZQ spectroscopy based on APSOC is proposed and the fundamental principles of $Z Q$ spectroscopy are formulated. This investigation opens a new way to obtain information on the sign of J-couplings using ZQ spectroscopy. The proposed method is also useful to track the redistribution of the singlet spin population in various PHIP related experiments, which is essential for efficient polarization transfer to target nuclei.
\end{abstract}

\section{Introduction}

Nuclear magnetic resonance (NMR) is a versatile technique that is used in many areas of science, medicine and technology. However, NMR suffers from low sensitivity which is caused by the weak interaction of a magnetic field with a nuclear magnetic moment being much smaller than the thermal energy, $k T$. The resulting small difference in the population of the spin energy levels, the nuclear spin polarization $P$, is directly proportional to the signal amplitude. At thermal equilibrium under ambient conditions and at a magnetic field of $10 \mathrm{~T}$ one gets only $P \approx 10^{-5}$; hence working off equilibrium can strongly boost the NMR signal. For this approach the term hyperpolarization has been coined. Various hyperpolarization techniques based on different physical and chemical effects have been developed. Particularly popular are dynamic nuclear polarization (DNP), ${ }^{1,2}$ optical pumping, ${ }^{3,4}$ chemically induced dynamic nuclear polarization (CIDNP) ${ }^{5}$ and para-hydrogen induced polarization (PHIP). ${ }^{6,7}$ Our work presented here was inspired by a recent contribution to the development of the PHIP hyperpolarization technique, namely photo initiated PHIP (photo-PHIP). ${ }^{8}$

All PHIP methods exploit the spin order of the para-spin isomer of $\mathrm{H}_{2}$ with its nuclei in the singlet state that is usually called para-hydrogen $\left(p \mathrm{H}_{2}\right)$. Due to its intrinsic symmetry $p \mathrm{H}_{2}$

\footnotetext{
${ }^{a}$ International Tomography Center SB RAS, Institutskaya 3a, Novosibirsk 630090, Russia.E-mail: a.n.pravdivtsev@gmail.com

${ }^{b}$ Novosibirsk State University, Pirogova 2, Novosibirsk 630090, Russia

${ }^{c}$ Nikolaev Institute of Inorganic Chemistry SB RAS, Acad. Lavrentiev Ave., 3, Novosibirsk 630090, Russia

${ }^{d}$ Institut für Experimentalphysik, Freie Universität Berlin, Arnimallee 14, Berlin 14195, Germany
}

is silent for NMR; hence, for making use of the huge nonequilibrium population the symmetry must be broken. In conventional PHIP experiments, the symmetry is broken in a catalytic hydrogenation reaction (usually $\mathrm{H}_{2}$ addition to a double or triple $\mathrm{C}-\mathrm{C}$ bond). As a result, the NMR signals of the pair-wise hydrogenation product are enhanced up to several orders of magnitude. ${ }^{9,10}$ Usually, for PHIP preparation $p \mathrm{H}_{2}$ is bubbled through the sample containing the reagent and catalyst, hence the preparation procedure is usually spread in time over several seconds. The classical PHIP example follows the protocol called parahydrogen and synthesis allow dramatically enhanced nuclear alignment (PASADENA). ${ }^{11}$ Although the starting $\mathrm{pH}_{2}$ is silent for NMR an anti-phased signal is observed in PASADENA because of the following reason: in the hydrogenation reaction the molecular Hamiltonian abruptly changes. The singlet spin state is not any more an eigen-state of the reagent, hence the wave-function starts oscillating. Since the reaction is distributed in time this oscillation dephases (loss of coherence due to the averaging). The system reaches its new eigen-states allowing one to obtain an observable signal.

In the conventional PHIP experiments the pure singlet state can be kept after hydrogenation only under the action of a strong RF-field, ${ }^{12}$ but in photo-PHIP experiments the singlet state is generated after a single laser shot with high purity. ${ }^{8}$ Current applications of pure singlet states are mainly optimization of generation of hyperpolarization in PHIP related experiments, ${ }^{13-15}$ and storage of hyperpolarization in long-lived spin states (LLS) ${ }^{16,17}$ and in long-lived coherences (LLC). ${ }^{18}$

In this contribution we are (1) giving an amendment to the interpretation of photo-PHIP experiments as it was given in the original paper, ${ }^{8}$ as well as (2) a generalization of the theoretical 
description of spin evolution of photo-PHIP in weakly coupled multi-spin systems ${ }^{19}$ that is confirmed by experimental observation. Using this theoretical approach we (3) formulate the rules for zeroquantum coherence spectroscopy (ZQCSy) in a weakly coupled spin system and illustrate them by experimental observation of the ZQ-spectrum of the AX pair in an $\mathrm{AXYZ}_{2}$ spin system. Finally (4) we suggest a way to investigate the evolution of coherent singlet spin states for any spin system without the need of photo initiation. While in the original photo-PHIP experiment the pure singlet state can be prepared only in rather specific photo excited metal complexes $^{8,19,20}$ our approach can be applied to arbitrary molecules and used for determination of the relative sign of $J$-couplings and for optimizing the strategy for generation of hyperpolarization, e.g. in PHIP related experiments.

\section{Results and discussion}

\subsection{Spin system}

For experimental demonstration and analysis we use the photoPHIP complex $\left[\mathrm{Ru}\left(\mathrm{PPh}_{3}\right)_{3}(\mathrm{CO})(\mathrm{H})_{2}\right]-1 .{ }^{8}$ Under the action of light excitation the $\mathrm{Ru}-\mathrm{HH}$ protons dissociate; as a result complex 2, $\left[\mathrm{Ru}\left(\mathrm{PPh}_{3}\right)_{3}(\mathrm{CO})\right]$, is formed (see Fig. 1). Complex 2 is transient, and shortly after $\mathrm{H}_{2}$ from the bulk binds to complex 2 to restore complex 1. When the solvent is saturated by $\mathrm{pH}_{2}$, then after photoactivation of complex 1 the non-polarized $\mathrm{Ru}-\mathrm{HH}$ protons are immediately (on the $\mu$ s timescale) substituted by $\mathrm{pH}_{2}$. Hence, as a result of light irradiation PHIP in complex $\mathbf{1}$ is generated. The relevant spin system of complex 1 is an $\mathrm{AXYZ}_{2}$ system (see Fig. 1) where A and $\mathrm{X}$ stands for the two $\mathrm{Ru}-\mathrm{HH}$ protons $\left(\mathrm{H}^{\mathrm{c}}\right.$ and $\left.\mathrm{H}^{\mathrm{t}}\right), \mathrm{Y}$ for the equatorial phosphorus $\left(\mathrm{P}^{\mathrm{e}}\right)$, and $\mathrm{Z}_{2}$ for the two axial phosphorus nuclei $\left(\mathrm{P}_{\mathrm{u}}^{\mathrm{a}}\right.$ and $\left.\mathrm{P}_{\mathrm{d}}^{\mathrm{a}}\right)$. Hereafter we assume that all spins are weakly coupled. The NMR parameters of this spin system are shown in Table 1.

\subsection{Sample preparation}

We synthesized complex 1 according to the protocol described in the literature. ${ }^{21}$ For all experiments shown here we used

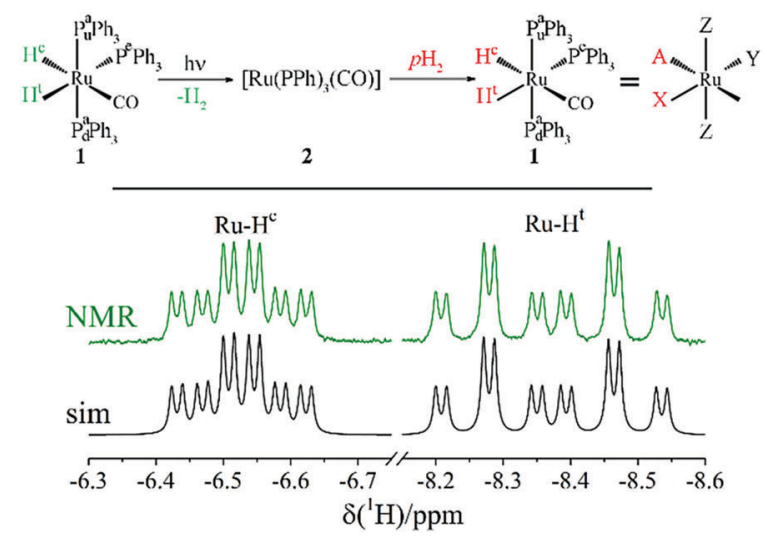

Fig. 1 Scheme of photo-PHIP generation resulting from photo-excitation of complex 1 in the presence of para-hydrogen $\left(\mathrm{pH}_{2}\right)$. Thermal NMR spectrum of the Ru-HH protons of complex 1 in toluene- $d_{8}$ at 9.4 Tesla (NMR) together with the simulated NMR spectrum using the parameters shown in Table 1 (sim).
Table 1 NMR parameters: chemical shifts, J-coupling constants for the $\mathrm{Ru}-\mathrm{HH}$ protons and ${ }^{31} \mathrm{P}$ of complex 1 ( $\mathrm{AXYZ}$, spin system)

\begin{tabular}{|c|c|c|c|c|c|c|}
\hline \multirow{2}{*}{\multicolumn{2}{|c|}{$J, \mathrm{~Hz}$}} & \multirow{2}{*}{$\frac{\mathrm{A}}{\mathrm{H}^{\mathrm{c}}}$} & \multirow{2}{*}{$\frac{\mathrm{X}}{\mathrm{H}^{\mathrm{t}}}$} & \multirow{2}{*}{$\frac{\mathrm{Y}}{\mathrm{P}^{\mathrm{e}}}$} & \multirow{2}{*}{$\frac{\mathrm{Z}}{\mathrm{P}_{\mathrm{u}}^{\mathrm{a}}}$} & \multirow{2}{*}{$\frac{\mathrm{Z}}{\mathrm{P}_{\mathrm{d}}^{\mathrm{a}}}$} \\
\hline & & & & & & \\
\hline $\mathrm{X}$ & $\mathrm{H}^{\mathrm{t}}$ & -6.5 & & & & \\
\hline Y & $\mathrm{P}^{\mathrm{e}}$ & -15.2 & 74.2 & & & \\
\hline $\mathrm{Z}$ & $\mathrm{P}_{\mathrm{u}}^{\mathrm{a}}$ & 30.7 & 28.4 & 17 & & \\
\hline $\mathrm{Z}$ & $\mathrm{P}_{\mathrm{d}}^{\mathrm{a}}$ & 30.7 & 28.4 & 17 & - & \\
\hline \multicolumn{2}{|c|}{$\delta, \mathrm{ppm}$} & -6.527 & -8.371 & 45.4 & 57.6 & \\
\hline
\end{tabular}

samples with $1 \mathrm{mM}$ concentration of complex 1 in toluene- $\mathrm{d}_{8}$. All photo-PHIP experiments were carried out on a Bruker $400 \mathrm{MHz}$ Avance III HD NMR spectrometer. The 2D ZQ spectrum shown in Fig. 10 was acquired on a Bruker $700 \mathrm{MHz}$ Avance III HD NMR spectrometer. For light excitation we used a Lambda Physik excimer laser with $308 \mathrm{~nm}$ wave length. The sample was irradiated from the side as described before. ${ }^{22}$

\subsection{Coherent singlet spin state in a two spin $\frac{1}{2}$ system}

As mentioned above the spin system of complex 1 consists of five nuclei: two protons ( $\mathrm{AX})$ and three phosphorus nuclei $\left(\mathrm{YZ}_{2}\right)$. Without loss of generality, let's first analyze the spin evolution of the two weakly coupled protons (AX) that are initially in the singlet spin state. And after that, all theoretical considerations will be compared with experimental observations of photoPHIP.

The scheme of the basic photo-PHIP experiment is shown in Fig. 2. At the beginning the sample is saturated by $p \mathrm{H}_{2}$. As a result of laser photo-excitation of $\mathbf{1}$ the non-polarized $\mathrm{Ru}-\mathrm{HH}$ protons are rapidly substituted by $\mathrm{pH}_{2}$ (for simplicity and without loss of generality let's assume that we use $100 \%$ enriched $p \mathrm{H}_{2}$ ). Hence, the initial state in our photo-PHIP experiment will be a pure singlet spin state $\hat{\rho}(\tau=-0)=\hat{\rho}_{\mathrm{S}}$. The first block of the pulse sequence $\left(p \mathrm{H}_{2}\right.$ bubbling and laser irradiation) can, in principle, be substituted by any pulse sequence for transfer of magnetization to singlet spin order $(\mathrm{M} 2 \mathrm{~S})^{23-25}$ to prepare the system in its pure singlet state $\hat{\rho}_{\mathrm{S}}$. This case will be discussed later in the text.

Now let's see what will happen with the singlet spin state during free evolution of the spin system. The Hamiltonian of two weakly coupled nuclei in a liquid is the following:

$$
\hat{H}_{0} / 2 \pi=-v_{1} \hat{I}_{1 \mathrm{Z}}-v_{2} \hat{I}_{2 \mathrm{Z}}+J \hat{I}_{1 \mathrm{Z}} \hat{I}_{2 \mathrm{Z}}
$$

where $v_{1,2}=\left(1+\delta_{1,2}\right) \gamma B / 2 \pi$ is the frequency of Zeeman interaction of the nuclei with the external magnetic field $B, J$ is the constant of scalar coupling, and $\delta_{i}$ is the chemical shift of the $i$ th nuclei.

It will be convenient for description of the spin dynamics to introduce and use the following terms of spin order:

(1) Zero-quantum coherence (ZQ) "in-phase":

$$
\mathrm{ZQX}=\hat{I}_{1 \mathrm{X}} \hat{I}_{2 \mathrm{X}}+\hat{I}_{1 \mathrm{Y}} \hat{I}_{2 \mathrm{Y}}
$$

(2) Zero-quantum coherence "out of phase":

$$
\mathrm{ZQ}_{\mathrm{Y}}=\hat{I}_{1 \mathrm{Y}} \hat{I}_{2 \mathrm{X}}-\hat{I}_{1 \mathrm{X}} \hat{I}_{2 \mathrm{Y}}
$$



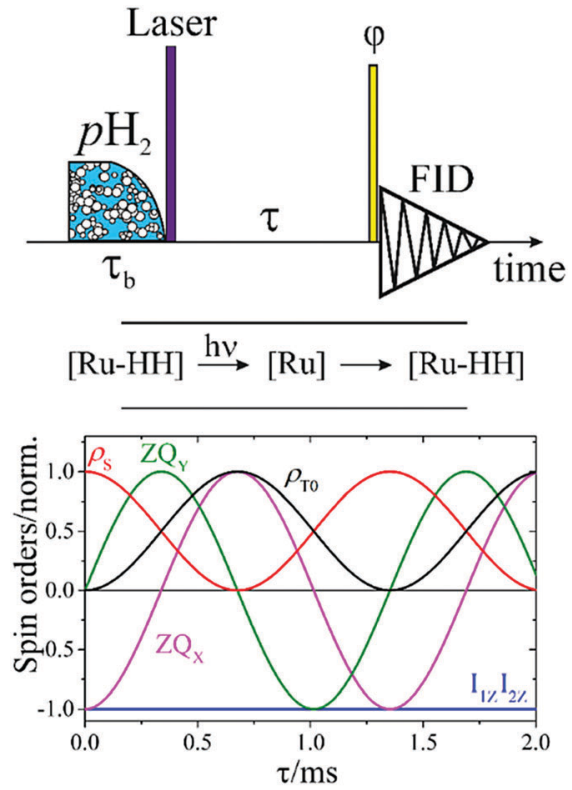

Fig. 2 (top) Basic photo-PHIP experimental scheme; (bottom) dependence of the following spin orders $\hat{l}_{1 Z} \hat{l}_{2 Z}, Z Q_{X}, Z Q_{Y}, \hat{\rho}_{S}$ and $\hat{\rho}_{T_{0}}$ on the time $\tau$ of free evolution in an AX spin system in a photo-PHIP experiment. Initially the system is in its pure singlet state $\hat{\rho}_{\mathrm{S}}$. The parameters of the AX system are as follows: $\delta_{1}=-6.47 \mathrm{ppm}, \delta_{2}=-8.3 \mathrm{ppm}, \mathrm{J}=-6.5 \mathrm{~Hz}$ corresponding to the parameters of the Ru- $\mathrm{HH}$ protons of complex 1 in toluene- $\mathrm{d}_{8}$. Here, the magnetic field is $9.4 \mathrm{~T}$.

(3) Density matrix of singlet state:

$$
\hat{\rho}_{\mathrm{S}}=E / 4-\left(\hat{\boldsymbol{I}}_{1}, \hat{\boldsymbol{I}}_{2}\right)=E / 4-I_{1 \mathrm{Z}} I_{2 \mathrm{Z}}-\mathrm{ZQ}_{\mathrm{X}}
$$

(4) Density matrix of $\mathrm{T}_{0}$ state:

$$
\hat{\rho}_{\mathrm{T}_{0}}=E / 4-\hat{I}_{1 \mathrm{Z}} \hat{I}_{2 \mathrm{Z}}+\mathrm{ZQ} \mathrm{X}
$$

The evolution of these spin orders is known and can be found in several sources. ${ }^{26-28}$

Under the impact of Zeeman interaction the $E / 4-\hat{I}_{1 \mathrm{Z}} \hat{I}_{2 \mathrm{Z}}$ term of $\hat{\rho}_{\mathrm{S}}$ does not evolve, while the $-\mathrm{ZQ}_{\mathrm{X}}$ coherence evolves in the following way:

$$
-\mathrm{Z} \mathrm{Q}_{\mathrm{X}} \stackrel{\pi \delta v \tau\left(\hat{I}_{1 \mathrm{Z}}-\hat{I}_{2 \mathrm{Z}}\right)}{\longrightarrow}-\mathrm{ZQ}_{\mathrm{X}} \cos (2 \pi \delta v \tau)+\mathrm{ZQ}_{\mathrm{Y}} \sin (2 \pi \delta v \tau)
$$

Hence, the evolution of the singlet spin state of the two-spin system can be easily described in terms of multiplet polarization, $\frac{E}{4}-\hat{I}_{1 \mathrm{Z}} \hat{I}_{2 \mathrm{Z}}$, and zero-quantum coherences, $\mathrm{ZQ}$ X and $\mathrm{ZQ}$, that oscillate with a frequency equal to the chemical shift difference $\delta v=\left|\nu_{1}-\nu_{2}\right|$. In some cases it is more convenient to track the evolution of populations, but not of coherences as will be shown in the text. The evolution of $\hat{\rho}_{\mathrm{S}}$ in terms of population of the $S$ and $\mathrm{T}_{0}$ states and the coherences between them is expressed in the following way:

$$
\begin{aligned}
& \hat{\rho}_{\mathrm{S}} \stackrel{\pi \delta v \tau\left(\hat{I}_{1 \mathrm{Z}}-\hat{I}_{2 \mathrm{Z}}\right)}{\longrightarrow} \cos ^{2}(\pi \delta v \tau) \hat{\rho}_{\mathrm{S}}+\sin ^{2}(\pi \delta v \tau) \hat{\rho}_{\mathrm{T} 0} \\
& +i / 2\left(|\mathrm{~S}\rangle\left\langle\mathrm{T}_{0}|-| \mathrm{T}_{0}\right\rangle\langle\mathrm{S}|\right) \sin (\pi \delta v \tau)
\end{aligned}
$$

Hence, the population of $\hat{\rho}_{\mathrm{S}}$ goes via $\mathrm{S}-\mathrm{T}_{0}$ coherences to the population of $\hat{\rho}_{\mathrm{T}_{0}}$ and back with the frequency $\delta v$. A numerical computation of the free evolution of the $\mathrm{ZQ}_{\mathrm{X}}, \mathrm{ZQ}_{\mathrm{Y}}, \hat{I}_{1 \mathrm{Z}} \hat{I}_{2 \mathrm{Z}}, \hat{\rho}_{\mathrm{S}}$ and $\hat{\rho}_{\mathrm{T}_{0}}$ terms of the two-spin system is shown in Fig. 2 .

Hence, in the photo-PHIP NMR spectrum the following three components contribute: (1) multiplet spin order, $E / 4-\hat{I}_{1 \mathrm{Z}} \hat{I}_{2 \mathrm{Z}}$, (2) zero-quantum coherence "in phase", ZQ $Q_{X}$, and (3) zeroquantum coherence "out of phase", $\mathrm{ZQ}_{\mathrm{Y}}$. All terms of the density matrix contribute differently to the observed NMR spectrum. Hence, due to evolution of the singlet spin state the shape of the NMR spectrum in photo-PHIP depends on the time span, $\tau$, between generation of the pure singlet state and the moment of registration. Let's see how the different spin-orders depend on the flip angle, $\varphi$, of a hard RF-pulse applied before acquisition of the free induction decay:

$$
\begin{aligned}
& \hat{I}_{1 \mathrm{Z}}+\hat{I}_{2 \mathrm{Z}} \stackrel{\varphi\left(\hat{I}_{1 \mathrm{X}}+\hat{I}_{2 \mathrm{X}}\right)}{\longrightarrow}-\frac{i}{2}\left(\hat{I}_{1-}+\hat{I}_{2-}\right) \sin (\varphi) \ldots \\
& \hat{I}_{1 \mathrm{Z}} \hat{I}_{2 \mathrm{Z}} \stackrel{\varphi\left(\hat{I}_{\mathrm{X}}+\hat{I}_{2 \mathrm{X}}\right)}{\longrightarrow}-\frac{1}{2} \frac{i}{2}\left(\hat{I}_{1-} \hat{I}_{2 \mathrm{Z}}+\hat{I}_{1 \mathrm{Z}} \hat{I}_{2-}\right) \sin (2 \varphi) \ldots \\
& \mathrm{ZQ}_{\mathrm{X}} \stackrel{\varphi\left(\hat{I}_{1 \mathrm{X}}+\hat{I}_{2 \mathrm{X}}\right)}{\longrightarrow} \frac{1}{2} \frac{i}{2}\left(\hat{I}_{1-} \hat{I}_{2 Z}+\hat{I}_{1 Z} \hat{I}_{2-}\right) \sin (2 \varphi) \ldots \\
& \mathrm{ZQ}_{\mathrm{Y}} \stackrel{\varphi\left(\hat{I}_{1 \mathrm{X}}+\hat{I}_{2 \mathrm{X}}\right)}{\longrightarrow} \frac{1}{2}\left(\hat{I}_{1 \mathrm{Z}} \hat{I}_{2-}-\hat{I}_{1-} \hat{I}_{2 \mathrm{Z}}\right) \sin (\varphi) \ldots
\end{aligned}
$$

Only " -1 " single quantum coherences are kept here $\left(\hat{I}_{1-}, \hat{I}_{1-} \hat{I}_{2 \mathrm{Z}}\right.$, etc. $)$ because it is agreed that they give rise to the NMR signal. ${ }^{26,27}$ From formulas (3) one can conclude the following:

(1) The amplitudes of the "out of phase" zero-quantum coherence, $\mathrm{ZQ}_{\mathrm{Y}}$, and of the longitudinal magnetization, $\hat{I}_{1 \mathrm{Z}}+\hat{I}_{2 \mathrm{Z}}$, depend on the detection angle $\varphi$ as $\sin (\varphi)$.

(2) The amplitudes of the "in phase" zero-quantum coherence, $\mathrm{ZQ}$, and of the multiplet polarization, $\hat{I}_{1 \mathrm{Z}} \hat{I}_{2 \mathrm{Z}}$, depend on the registration angle $\varphi$ as $\sin (2 \varphi)$.

(3) If one uses a registration pulse with angle $\varphi=\pi / 2$ only $\mathrm{ZQ}_{\mathrm{Y}}$ (and $\hat{I}_{1 \mathrm{Z}}+\hat{I}_{2 \mathrm{Z}}$ if present) contributes to the NMR spectrum, i.e. $\hat{I}_{1 \mathrm{Z}} \hat{I}_{2 \mathrm{Z}}$ and $\mathrm{ZQ}$ are filtered out.

(4) The amplitude of the NMR spectrum of $Z_{Y}$ recorded with a $(\pi / 2)$ RF-pulse is twice the amplitude of the NMR spectrum of $\mathrm{ZQ}_{\mathrm{X}}$ or $\hat{I}_{1 \mathrm{Z}} \hat{I}_{2 \mathrm{Z}}$ recorded with a $(\pi / 4)$ RF-pulse as conventionally used for detection in PHIP experiments.

In Fig. 3 the real and imaginary parts of NMR spectra from the already mentioned spin orders, $\hat{I}_{1 \mathrm{Z}} \hat{I}_{2 \mathrm{Z}}, \mathrm{ZQ}_{\mathrm{X}}, \mathrm{ZQ_{ \textrm {Y } }}$ and $\hat{I}_{1 \mathrm{Z}}+\hat{I}_{2 \mathrm{Z}}$, are shown for comparison and illustration of the above-mentioned points. Here, two registration angles using $\left(45^{\circ}\right)$ and $\left(90^{\circ}\right)$ hard-RF pulses are applied. In the following subsection the theoretical predictions, which were obtained here for a two-spin $\frac{1}{2}$ system, will be illustrated by experimental observations on complex 1.

\subsection{Dependence of evolved singlet spin order on the registration angle}

To prove the theoretical consideration given above it is enough to show that there are only two components in the NMR spectrum of a system that starts from a pure singlet spin state: 

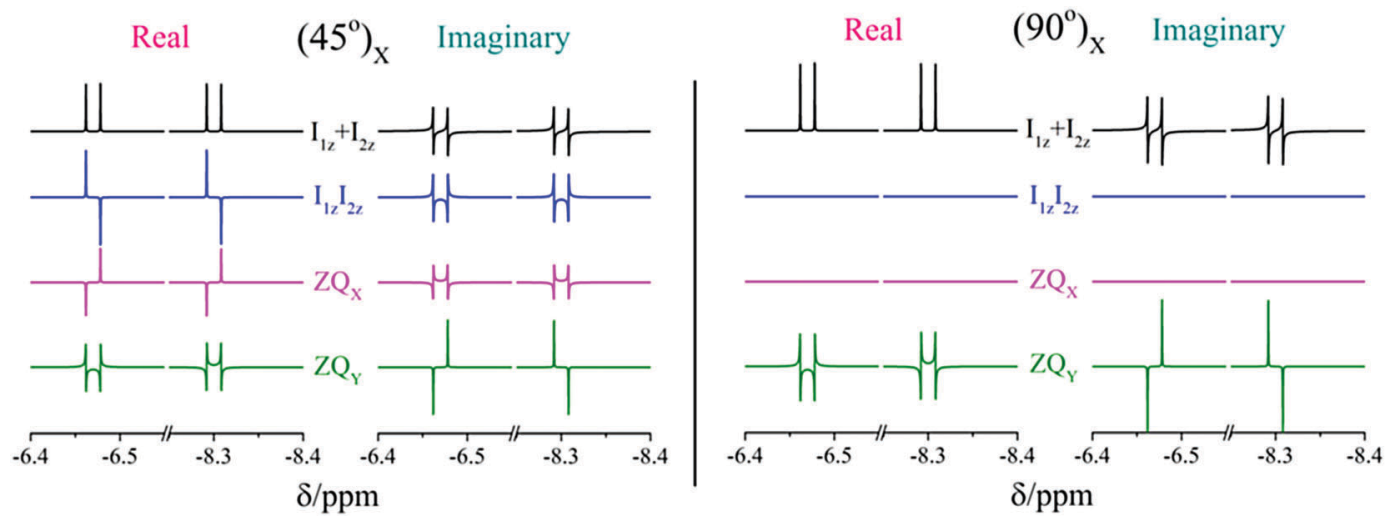

Fig. 3 Calculated NMR spectra of the AX system of the Ru-HH protons in complex 1 for four different spin orders: (1) Z-magnetization, $\hat{l}_{1 Z}+\hat{l}_{2 Z}$, (2) multiplet polarization, $\hat{I}_{1 Z} \hat{l}_{2 Z}$, (3) zero-quantum coherence "in phase", ZQ $Q_{x}$ (4) zero-quantum coherence "out of phase", ZQY. All spectra are calculated for a hard RF-pulse with the $\left(45^{\circ}\right) \times$ (left) and $\left(90^{\circ}\right) \times$ (right) flip angle. The magnetic field is $9.4 \mathrm{~T}$.

$\mathrm{ZQ}_{\mathrm{Y}}$ and a superposition of $\mathrm{ZQ}_{\mathrm{x}}$ and $\hat{I}_{1 \mathrm{Z}} \hat{I}_{2 \mathrm{Z}}$. The strategy to deconvolve the dependence of different spectral patterns on the angle of a hard RF detection pulse is known. ${ }^{12,29}$ Here, we applied it for photo-PHIP complex $\mathbf{1}$. We recorded 32 photoPHIP NMR spectra after a free evolution delay of $\tau \sim 0.15 \mathrm{~ms}$ with the angle $\varphi_{k}=\pi k / 16, k=1,2, \ldots, 32$. Then we obtained the components of the Fourier series that depend on the rotation angle as $\sin (\varphi)(\mathrm{FS} 1)$ and $\sin (2 \varphi)$ (FS2). The experimental spectra together with simulated spectra of different spin orders of the Ru-HH protons of complex 1 are shown in Fig. 4. One can see an excellent agreement between experimental observations and theoretical predictions and compare the obtained results with the simulated spectra for the two $\frac{1}{2}$ spin system in Fig. 3 . $\mathrm{ZQ}$ differs from $\mathrm{ZQ}$ and $\hat{I}_{1 \mathrm{Z}} \hat{I}_{2 \mathrm{Z}}$ not only by its angular dependence but also by the spectral pattern. The "in-phase" (real part

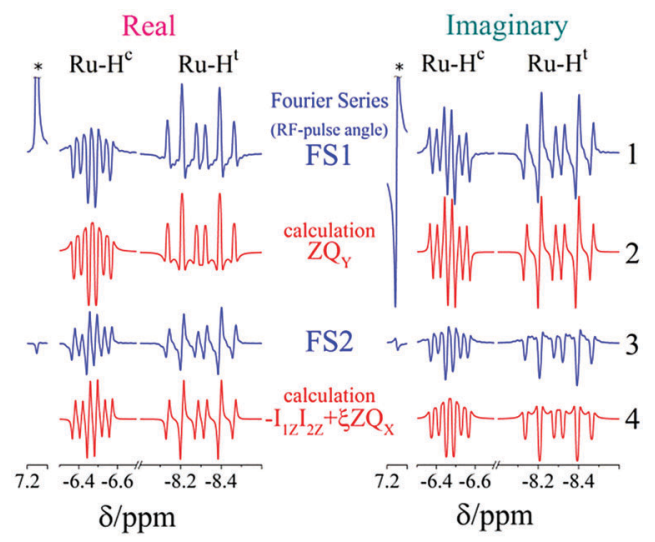

Fig. 4 Real and imaginary parts of two Fourier harmonics of the photoPHIP NMR spectrum obtained after a free evolution delay of $\tau \sim 0.15 \mathrm{~ms}$. The Fourier harmonics are taken with respect to the flip angle of the detection RF-pulse (FS1, FS2): spectrum 1 (FS1) corresponds to spin order that depends on the rotation angle $\varphi$, as $\sin (\varphi)$, spectrum 3 (FS2) - as $\sin (2 \varphi)$. Spectrum 2 is the computation of the NMR spectrum of "out of phase" zero-quantum coherence $Z Q_{Y}$, spectrum 4 corresponds to the superposition of spin order $-l_{1 Z} I_{2 Z}$ and $Z Q_{x}$. Higher harmonics were not observed experimentally. Asterisks indicate the thermally polarized signal from the solvent. The external magnetic field is 9.4 Tesla. of the spectrum) component of the $\mathrm{ZQ}_{\mathrm{Y}}$ spectrum has a dispersive pattern while the "in-phase" (real part of the spectrum) component of ZQx and $\hat{I}_{1 \mathrm{Z}} \hat{\mathrm{I}}_{2 \mathrm{Z}}$ has an anti-phase pattern. The imaginary parts of the spectra are vice versa. Hence the used simplification of the five-spin system by a two-spin system is valid and allows us to analytically explain the photo-PHIP spectral components and obtain them separately for the first time.

\subsection{Zero quantum beats and modulations}

As pointed out it is more advantageous to use a 90-degree registration RF-pulse, because we filter out the spectral contributions from the $I_{1 \mathrm{Z}} I_{2 \mathrm{Z}}$ and $\mathrm{ZQ}$ components, i.e. we detect only the spectrum from a single spin order, $\mathrm{ZQ}_{\mathrm{Y}}$. Using the photo-PHIP sequence shown in Fig. 2 we have tracked the free evolution of the coherent singlet state in complex 1 by observation of $\mathrm{ZQ}_{\mathrm{Y}}$. The dependence of the $\mathrm{ZQ}_{\mathrm{Y}}$ coherence on the free evolution time $\tau$ is shown in Fig. 5. An almost identical protocol was used in a previous article with the same complex: ${ }^{8}$ the ZQC oscillates due to the chemical shift difference of the two protons with an oscillation frequency of $\delta v \sim 738 \mathrm{~Hz}$ at a field of $9.4 \mathrm{~T}$; however, no modulations of the $\mathrm{ZQ}_{\mathrm{Y}}$ coherence were found before (compare Fig. 5(a) and 6(b) of ref. 8). In that publication the signal decay in the interval from 0 to $6 \mathrm{~ms}$ was fitted by an exponentially decaying sine function ${ }^{8}$ and tentatively attributed to the decay by relaxation processes. In contrast, we found that the oscillations in the evolution of $\mathrm{ZQ}_{\mathrm{Y}}$ are proceeding much longer showing a modulation with the frequency $v_{\mathrm{m}} \sim 46 \mathrm{~Hz}$ (see Fig. 5(a)) and measured the damping time of the evolution by sampling the oscillation at intervals $\tau_{k}=(k+1 / 4) / \delta v$ with $k$ belonging to natural numbers. The kinetics is shown in Fig. 5(b). From the decay of the modulation we can now estimate the lifetime of the zero quantum coherence to be not less than $0.15 \mathrm{~s}$. The Fourier transformation of the 1D spectra in the indirect dimension gives us the 2D spectrum shown in Fig. 5(c). It is seen that for each of the Ru-HH protons the triplets corresponding to a different spin projection of the equatorial phosphorus nuclei have a different resonance frequency $\delta v \pm v_{\mathrm{m}}$ in the indirect dimension.

These modulations are not predicted by the oversimplified two-spin system and can be explained only if one includes all 


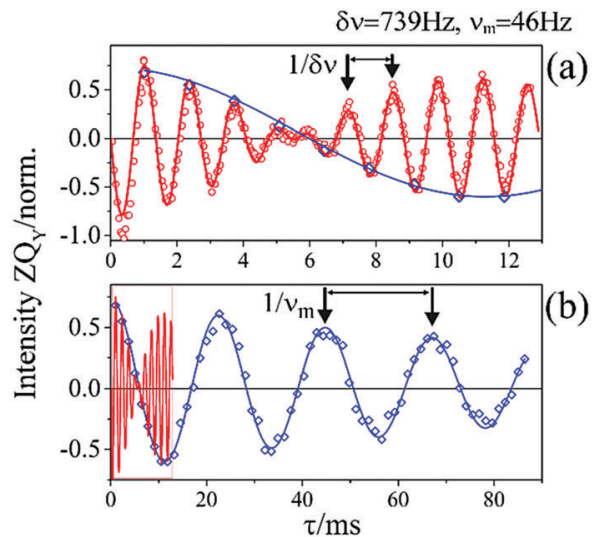

(c)

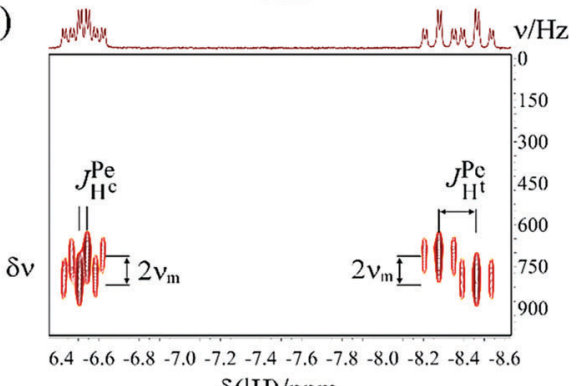

$\delta(' \mathrm{H}) / \mathrm{ppm}$

Fig. 5 Evolution of the "out of phase" zero-quantum coherence, $Z Q_{Y}$, in the photo-PHIP experiment for complex 1. (a) Fast oscillations are shown (red circles) with their frequency equal to the chemical shift difference $\delta v=v_{\mathrm{H}^{c}}-v_{\mathrm{H}^{\mathrm{t}}}=737.7 \mathrm{~Hz}$ and compared to a numerical computation (red solid line). (b) The modulation curve is shown (blue diamonds), as well as the fitting function $f(\tau)=A \cos \left(2 \pi v_{m} \tau\right) e^{-\tau / T}$ with frequency $v_{m}=44 \mathrm{~Hz}$ and characteristic damping time $T=0.15 \mathrm{~s}$. (c) Fourier transformation in the indirect dimension for the series of 1D spectra analyzed in (a). The external magnetic field is 9.4 Tesla.

other interactions in the spin system. In our case the main term is coming from the three phosphorus nuclei. At first let's track the evolution of zero-quantum coherences in an AXY spin system (here, the additional $\mathrm{Y}$ nucleus represents a single phosphorus in our spin system). Evolution of zero and multiple quantum coherences in general cases was investigated before; ${ }^{28}$ here we will only give the derivation of spin evolution relevant for us. Under the action of scalar coupling with spin $\mathrm{Y}$ the $\mathrm{ZQ}_{\mathrm{x}}$ component of the AX pair will evolve in the following way:

$$
\begin{aligned}
& \mathrm{ZQ}_{\mathrm{X}} \stackrel{2 \pi J_{\mathrm{A}}^{\mathrm{Y}} \hat{I}_{\mathrm{Z} Z} \hat{I}_{3 Z}}{\longrightarrow} \mathrm{ZQ}_{\mathrm{X}} \cos \left(\pi J_{\mathrm{A}}^{\mathrm{Y}} \tau\right)+2 \mathrm{ZQ}_{\mathrm{Y}} \hat{I}_{3 \mathrm{Z}} \sin \left(\pi J_{\mathrm{A}}^{\mathrm{Y}} \tau\right) \\
& \stackrel{2 \pi J_{\mathrm{X}}^{\mathrm{Y}} \hat{I}_{1 Z} \hat{I}_{3 Z}}{\longrightarrow} \mathrm{ZQ} \mathrm{Q}_{\mathrm{X}} \cos \left(\pi \Delta J_{\mathrm{AX}}^{\mathrm{Y}} \tau\right)+2 \mathrm{ZQ}_{\mathrm{Y}} \hat{I}_{3 \mathrm{Z}} \sin \left(\pi J_{\mathrm{AX}}^{\mathrm{Y}} \tau\right)
\end{aligned}
$$

and $\mathrm{ZQ}_{\mathrm{Y}}$ in the following way:

$$
\begin{aligned}
& \mathrm{ZQ}_{\mathrm{Y}} \stackrel{2 \pi J_{\mathrm{A}}^{\mathrm{Y}} \hat{I}_{1 Z} \hat{I}_{3 Z}}{\longrightarrow} \mathrm{ZQ}_{\mathrm{Y}} \cos \left(\pi J_{\mathrm{A}}^{\mathrm{Y}} \tau\right)-2 \mathrm{ZQ}_{\mathrm{X}} \hat{I}_{3 \mathrm{Z}} \sin \left(\pi J_{\mathrm{A}}^{\mathrm{Y}} \tau\right) \\
& \stackrel{2 \pi J_{\mathrm{X}}^{\mathrm{Y}} \hat{I}_{1 Z} \hat{I}_{3 Z}}{\longrightarrow} \mathrm{ZQ} \mathrm{Q}_{\mathrm{Y}} \cos \left(\pi \Delta J_{\mathrm{AX}}^{\mathrm{Y}} \tau\right)-2 \mathrm{Z} \mathrm{Q}_{\mathrm{Y}} \hat{I}_{3 \mathrm{Z}} \sin \left(\pi J_{\mathrm{AX}}^{\mathrm{Y}} \tau\right)
\end{aligned}
$$

where $\Delta J_{\mathrm{AX}}^{\mathrm{Y}}=J_{\mathrm{A}}^{\mathrm{Y}}-J_{\mathrm{X}}^{\mathrm{Y}}$. It follows that all additional nuclei will cause modulation of $\mathrm{ZQ}_{\mathrm{X}}$ and $\mathrm{ZQ}_{\mathrm{Y}}$ coherences with the frequency equal to half the difference in $J$-coupling of the $\mathrm{Ru}-\mathrm{HH}$ protons with the additional nucleus, $\Delta J_{\mathrm{AX}}^{\mathrm{Y}} / 2$. And indeed, we observed such modulation in our photo-PHIP experiment (see $\mathrm{ZQ}_{\mathrm{Y}}$ modulations in Fig. 5(a) and splitting of NMR peaks in the indirect dimension in Fig. 5(c)). The same splitting in 2D spectra was recently found and explained for AXY and AXYZ spin systems; ${ }^{19}$ however, the original experiments and their explanation ${ }^{8}$ were not discussed.

To model the free evolution of the pure singlet spin state in arbitrarily coupled spin systems (up to 14 spins) we wrote software which is available online. ${ }^{30}$ Our software calculates the evolution of $\mathrm{ZQX}_{\mathrm{X}}, \mathrm{ZQ}_{\mathrm{Y}}, \rho_{\mathrm{S}}$ and $\rho_{\mathrm{T}_{0}}$ spin orders when a pair of nuclei starts from a pure singlet state. Relaxation is taken into account using the Redfield theory of relaxation with fluctuating local magnetic fields. ${ }^{31,32}$ If one takes the Fourier transformation over $\mathrm{ZQ}_{\mathrm{X}}+i \mathrm{ZQ}_{\mathrm{Y}}$ (free $\mathrm{ZQ}$ decay) spectra analogous to those shown in Fig. 6 are obtained. In Fig. 6 we demonstrate the effect of extra nuclei on the evolution of the pure singlet spin state of the $\mathrm{AX}$ pair in $\mathrm{AX}, \mathrm{AXY}, \mathrm{AXYZ}$ and $\mathrm{AXYZ}_{2}$ spin systems. The spectrum for the $\mathrm{AXYZ}_{2}$ spin system corresponds to the splitting of the 2D NMR spectrum in the indirect dimension shown in Fig. 5(c). In our experiment the extra splitting due to interaction with two axial phosphorus nuclei was not observed because photo-PHIP system 1 degrades after several tens of laser shots; therefore it was not feasible to record the necessary number of data. Below we will show how one can obtain the same 2D ZQ spectrum for an arbitrary spin system without photo-excitation.

It is interesting that the spectra from the free ZQC decay resemble normal NMR spectra that are in fact spectra from a free single quantum coherence decay. In summary, the rules for explanation of spectra from ZQCs in weakly coupled spin systems are the following:

(1) The resonance frequency in the ZQ spectrum of a spin pair A and X is their chemical shift difference: $\delta v=\left|v_{\mathrm{A}}-v_{\mathrm{X}}\right|$.

(2) The structure of all multiplets is the same as in standard NMR spectra, only that here the splitting is given by the

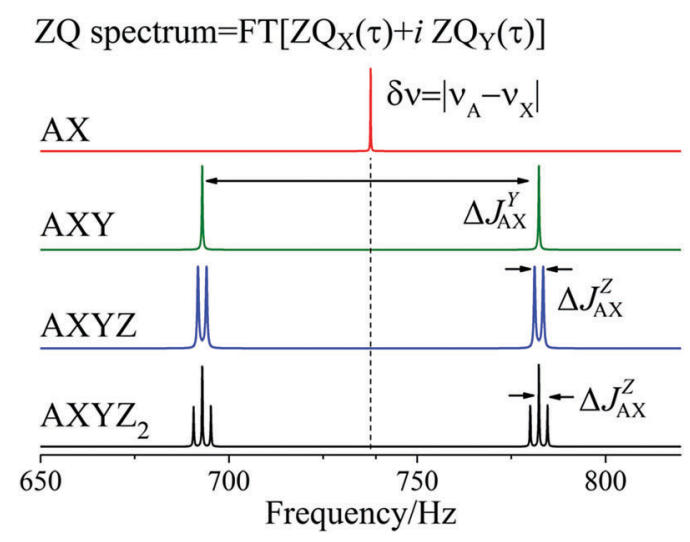

Fig. 6 Fourier transformation of $Z Q_{X}(\tau)+i Z Q_{Y}(\tau)$ in a photo-PHIP experiment for $A X, A X Y, A X Y Z$ and $A X Y Z_{2}$ spin systems. Here $\Delta J_{A X}^{Y}=\left|J_{A}^{Y}-J_{X}^{Y}\right|$, $\Delta J_{A X}^{Z}=\left|J_{A}^{Z}-J_{X}^{Z}\right|, \delta v=\left|v_{A}-v_{X}\right|$ and $T_{1}=T_{2}=5 \mathrm{~s}$ for all nuclei and a magnetic field of 9.4 T. NMR parameters are taken from Table 1. 
difference in $J$-coupling with other nuclei, e.g. the splitting of the ZQ spectrum of the AX pair interacting with nucleus $\mathrm{Y}$ is given by $\Delta J_{\mathrm{AX}}^{\mathrm{Y}} \rightarrow\left|J_{\mathrm{A}}^{\mathrm{Y}}-J_{\mathrm{X}}^{\mathrm{Y}}\right|$.

From the conventional NMR spectrum of weakly coupled spin systems (see Fig. 1) we are able to determine the absolute values of all $J$-couplings. From the splitting in the 1D ZQC spectrum (see Fig. 6) or in the 2D ZQ spectrum (see Fig. 5(c)) we can obtain the absolute value of the difference in $J$-coupling. Using all information explained above we concluded the following:

(1) Because $2 v_{\mathrm{m}} \sim \Delta J_{\mathrm{HH}}^{\mathrm{Pe}}=\left|J_{\mathrm{H}^{\mathrm{c}}}^{\mathrm{Pe}}-J_{\mathrm{H}^{\mathrm{t}}}^{\mathrm{Pe}}\right|=89.4 \mathrm{~Hz} \quad$ and $\left|J_{\mathrm{H}^{\mathrm{c}}}^{\mathrm{Pe}^{\mathrm{e}}}\right|=15.2 \mathrm{~Hz}$ it follows that $\operatorname{sign}\left(J_{\mathrm{H}^{\mathrm{t}}}^{\mathrm{Pe}^{\mathrm{e}}}\right)=-\operatorname{sign}\left(J_{\mathrm{H}^{\mathrm{t}}}^{\mathrm{Pe}}\right)$,

(2) Because $\left|J_{\mathrm{H}^{\mathrm{c}}}^{\mathrm{pa}}\right|=30.7 \mathrm{~Hz},\left|J_{\mathrm{H}^{\mathrm{t}}}^{\mathrm{pa}}\right|=28.4 \mathrm{~Hz}$ and the absence of any observable splitting of the order of $\left|J_{\mathrm{H}^{\mathrm{a}}}^{\mathrm{\textrm {P } ^ { \mathrm { a } }}}\right|+\left|J_{\mathrm{H}^{\mathrm{a}}}^{\mathrm{p}}\right| \sim 60 \mathrm{~Hz}$, we conclude that $\Delta J_{\mathrm{HH}}^{\mathrm{Pa}}=\left|J_{\mathrm{H}^{\mathrm{c}}}^{\mathrm{Pa}}\right|-\left|J_{\mathrm{H}^{\mathrm{t}}}^{\mathrm{P}^{\mathrm{a}}}\right|=2.3 \mathrm{~Hz} \ll 60 \mathrm{~Hz}$ and $\operatorname{sign}\left(J_{\mathrm{H}^{\mathrm{c}}}^{\mathrm{pa}}\right)=\operatorname{sign}\left(J_{\mathrm{H}^{\mathrm{t}}}^{\mathrm{Pa}}\right)$,

Spectra with better resolution in the indirect dimension will be shown below that will prove the abovementioned conclusions by direct observation of multiplet splittings in the $2 \mathrm{D}$ ZQC spectrum with the value $\Delta J_{\mathrm{HH}}^{\mathrm{pa}} \sim 2.3 \mathrm{~Hz}$ due to interactions of two protons with the axial phosphorus.

In summary, we have shown that analysis of ZQCs can be helpful to characterize the spin system and determine e.g. relative signs of $J$-couplings; however, it is rather inconvenient to analyze anti-phase and dispersive multiplets from $\mathrm{ZQ}_{\mathrm{X}}$ and $\mathrm{ZQ}_{\mathrm{Y}}$ as shown in Fig. 4. In the following part of our article we describe how one can directly follow the population of a singlet spin state and observe in-phase spectra instead of anti-phase spectra.

\subsection{S2M photo-PHIP detection scheme}

The straightforward approach to investigate photo-PHIP does not allow one to follow the evolution of a singlet spin state directly since it is unobservable in NMR. However, one can transfer singlet spin population to magnetization (S2M) using techniques well described in the literature. ${ }^{33-35}$ Here we choose the adiabatic passage spin-order conversion technique (APSOC) ${ }^{23}$ because of its generality and robustness in application. The experimental photo-PHIP protocol with APSOC modification is shown in Fig. 7. An appropriately set adiabatic RF-field ramp transfers population of the singlet state into population of the triplet state as schematically shown in Fig. 7.

The result of such an application of the APSOC sequence to photo-PHIP is shown in Fig. 8(a). The sign of magnetization of the Ru-HH protons depends on the frequency, $v_{\mathrm{rf}}$, of the RF-field during the APSOC stage. The corresponding frequency dependence (experimental and modeled) is shown in Fig. 8(b). By using a "phase-frequency" cycle ${ }^{36}$ one can subtract the background signal from thermally polarized species (see Fig. 8(a)), a procedure that is termed singlet order selection (SOS)-filter. ${ }^{36}$ The evolution of singlet spin population, $\rho_{\mathrm{S}}$, proceeds as the $[1+\cos (2 \pi \delta v \tau)] / 2$ function as predicted by eqn (2) (see and compare Fig. 2 and $8(\mathrm{c})$ ). We observe the same features, an oscillation given by the chemical shift difference, $\delta v$, as in the $\mathrm{ZQ}_{\mathrm{Y}}$ coherence (see Fig. 5) and modulations due to $J$-couplings with phosphorus nuclei with frequency $\Delta J_{\mathrm{HH}}^{\mathrm{pe}} / 2$.

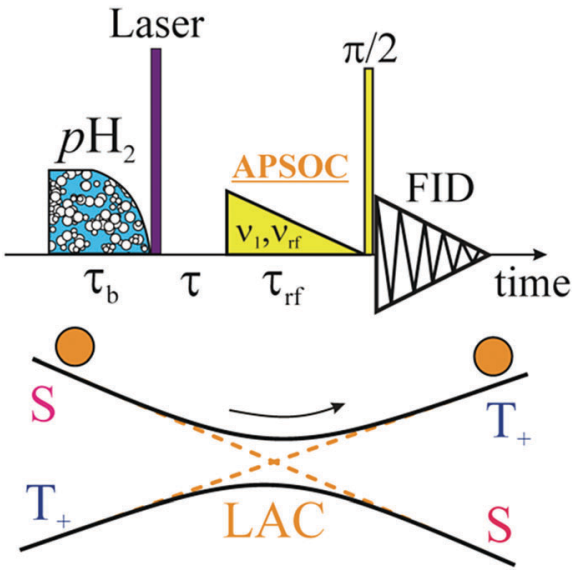

Fig. 7 (top) Protocol of photo-PHIP experiment with the APSOC block (fading RF-field of duration $\tau_{\mathrm{rf}}$ with frequency $v_{\mathrm{rf}}$, and initial amplitude $v_{1}^{0}$ ) that transfers population of the singlet state into population of the $T_{+}$(or $T_{-}$) state. (bottom) Level anti-crossing (LAC) diagram during the APSOC stage. Here, balls represent populations of spin states.
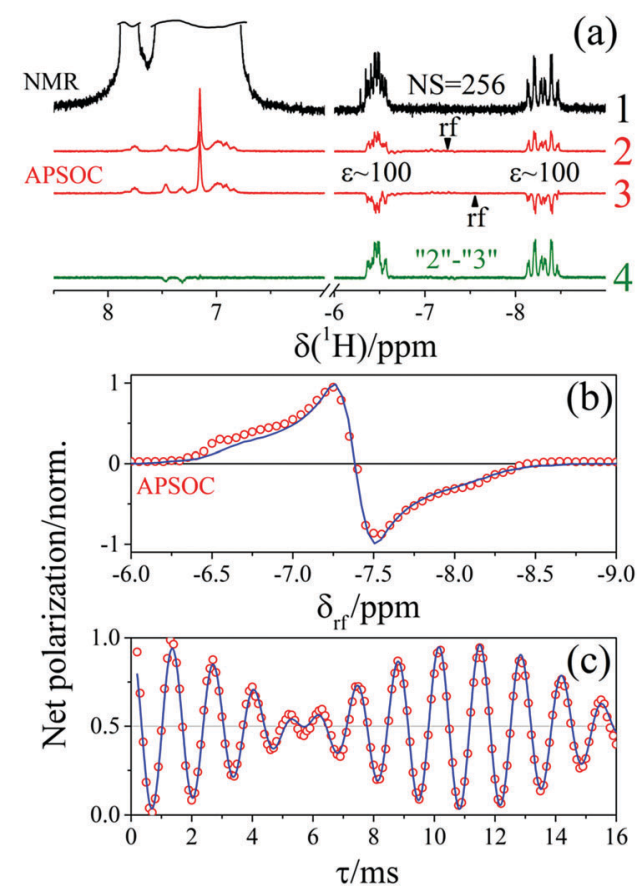

Fig. 8 (a) NMR spectra of complex 1 in toluene- $d_{8}$ : (spectrum 1) thermal NMR spectrum obtained after the accumulation of 256 transients; (spectra 2 and 3) photo-PHIP spectra obtained using APSOC with a linear RF-field profile during the time period $\tau_{\mathrm{rf}}=0.2 \mathrm{~s}$ and an initial amplitude $v_{1}=6 \mathrm{kHz}$; RF-frequency, $v_{\mathrm{rf}}$, is $-7.25 \mathrm{ppm}$ and $-7.55 \mathrm{ppm}$, respectively; (spectrum 4) the difference of spectra 2 and 3. (b) Frequency dependence of photoPHIP on Net-magnetization conversion efficiency. Experimental measurements are shown by circles, while the blue solid line represents simulation. (c) $\tau$-Dependence of net polarization in photo-PHIP APSOC experiment. The oscillation frequency here is $737.7 \mathrm{~Hz}$, and modulation is about $44 \mathrm{~Hz}$. Enhancements of $\mathrm{Ru}-\mathrm{HH}$ signals with respect to the thermal signal of all $\mathrm{Ru}-\mathrm{HH}$ protons are about $\sim 100$ at $B_{0}=9.4$ Tesla.

It is advantageous to transfer singlet spin order to magnetization because: (1) a net polarized spectrum is easier to analyze and (2) the same information on spin interactions 


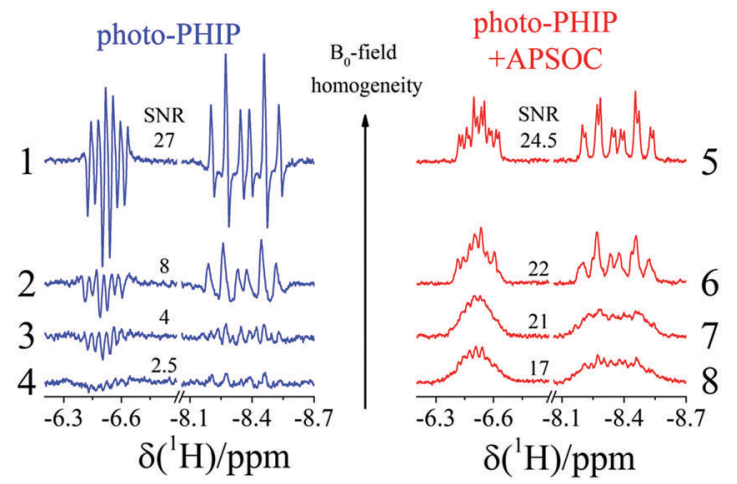

Fig. 9 Photo-PHIP spectra of complex 1 detected by a 90-degree RF-pulse (1-4) and photo-PHIP NMR spectra obtained using the APSOC M2S procedure (5-8). $B_{0}$ homogeneity was deliberately spoiled and it is the same in pairs of spectra 1 and 5,2 and 6,3 and 7 , and 4 and 8 . Average SNRs of the two ${ }^{1} \mathrm{H}$ signals are given. Homogeneity of $B_{0}$ increases from bottom to top.

can be obtained. Also, we want to draw attention to the importance of transferring multiplet polarization to magnetization to improve the SNR in case of insufficient $B_{0}$ homogeneity. For demonstration, we changed currents in the shim coils $(\mathrm{Z}$ and $\mathrm{X})$ to spoil homogeneity of the $B_{0}$ magnetic field. You can compare the SNR of photo-PHIP spectra detected by a simple 90-degree RF-pulse with detection after the APSOC S2M conversion procedure (see Fig. 9). While the SNR of the photo-PHIP signal decreases by a factor of 10 the SNR of the photo-PHIP + APSOC S2M block decreases only by 30\%! Hence, the advantages of using S2M conversion techniques are obvious, and we suggest using it in future work not only with photo-PHIP, but also in conventional high field PHIP experiments.

\subsection{D ZQ spectra using Boltzmann polarization}

If there is no problem with NMR-sensitivity it is sometimes advantageous to use thermal polarization because, e.g., there is no degradation of the complex due to light irradiation. The APSOC S2M sequence can be used backwards to transfer magnetization to singlet spin order (M2S). We suggest the scheme shown in Fig. 10(a) to obtain 2D ZQ spectra that will give the same amount of information as $2 \mathrm{D}$ photo-PHIP experiments; however, this sequence can be applied to any molecule of choice and to any coupled homonuclear spin pairs. Here we applied it to the same complex 1. The 2D ZQ sequence consists of a M2S block; in our case it is a rising linear RF-field with frequency $v_{\mathrm{rf}}=-7.25 \mathrm{ppm}$ or $v_{\mathrm{rf}}=-7.55 \mathrm{ppm}$, duration $\tau_{\text {rf }}=0.2 \mathrm{~s}$ and final amplitude $v_{1}=6 \mathrm{kHz}$. To filter out background signals we used the same "frequency-phase" cycle as in an SOS-filter. ${ }^{36}$ Then the system freely evolves during the time $\tau$ and finally is detected using an appropriate registration scheme. Here we applied a 90-degree pulse as in the photo-PHIP experiment (compare the 2D ZQ protocol with the photo-PHIP protocol in Fig. 2). We also applied ${ }^{31} \mathrm{P}$ decoupling during the M2S stage to improve the performance of the APSOC procedure.

We obtained $4 \mathrm{k}$ transients with dwell time $0.00012 \mathrm{~s}$ (without quadrature detection in the indirect dimension) that
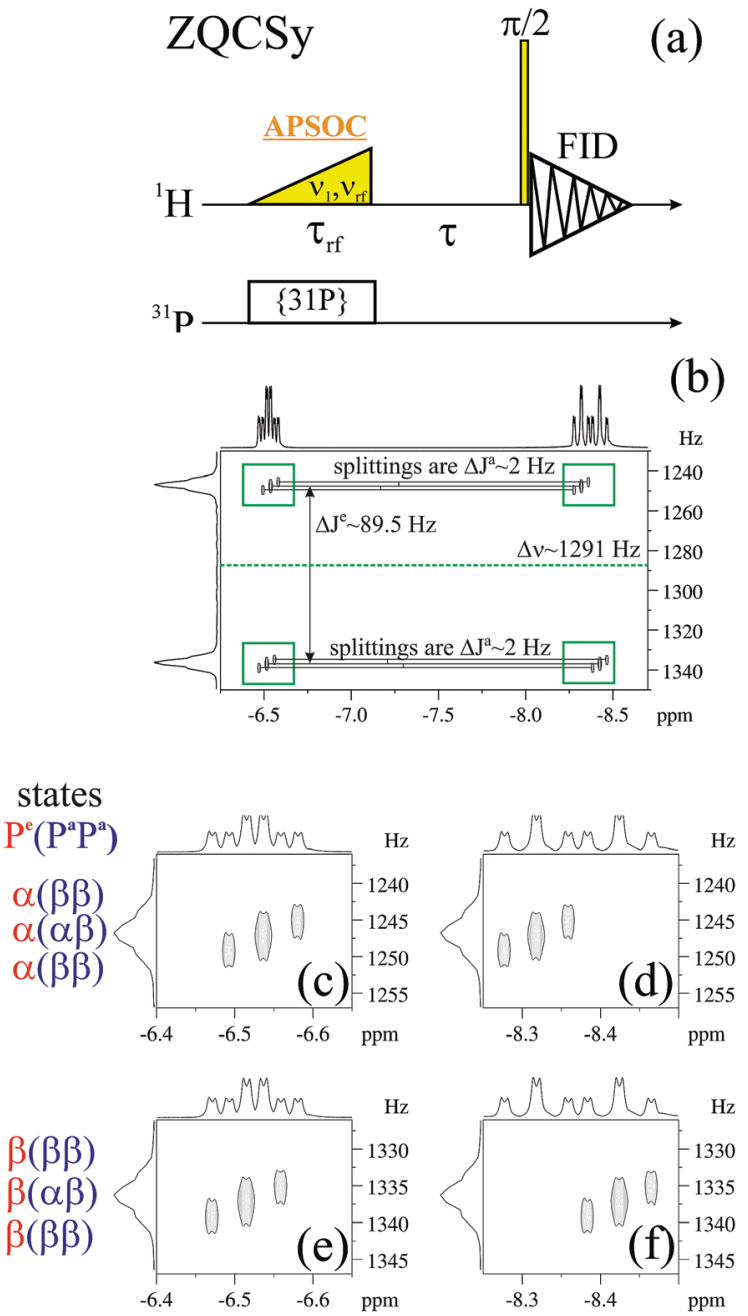

Fig. 10 (a) 2D ZQ sequence. Here, for the M2S step APSOC is applied together with ${ }^{31} \mathrm{P}$ decoupling. (b) $2 \mathrm{D} Z \mathrm{ZQ}$ spectrum of 1 . Here, $4 \mathrm{k}$ transients are acquired. All couplings with phosphorus nuclei are observable. $\Delta J_{\mathrm{HH}}^{\mathrm{pe}}=\left|J_{\mathrm{H}^{\mathrm{c}}}^{\mathrm{pe}}-J_{\mathrm{H}^{\mathrm{t}}}^{\mathrm{Pe}}\right|=89.5 \mathrm{~Hz}, \quad \Delta J_{\mathrm{HH}}^{\mathrm{pa}}=\left|J_{\mathrm{H}^{\mathrm{c}}}^{\mathrm{pa}}-J_{\mathrm{H}^{\mathrm{t}}}^{\mathrm{a}}\right|=2.3 \mathrm{~Hz}$ and $\delta v=$ $V_{H^{c}}-V_{H^{t}}=1291 \mathrm{~Hz}$. (c-f) Expansions of the marked regions in spectrum (b). Spin states of phosphorus nuclei that correspond to different resonance frequencies in the indirect dimension are given. The external magnetic field is 16.4 Tesla.

corresponds to the following parameters in the indirect dimension: acquisition time $\sim 0.5 \mathrm{~s}$, spectral width $\sim 8.3 \mathrm{kHz}$ and spectral resolution $2 \mathrm{~Hz}$. We deliberately acquired enough points to digitize the fastest frequency $\delta v \sim 1.2 \mathrm{kHz}$ and obtain spectral resolution in the indirect dimension of $c a .2 \mathrm{~Hz}$ to demonstrate the 2D ZQ spectrum without aliasing. Due to the degradation of complex 1 under light exposure it is impossible to achieve such a high resolution in a photo-PHIP experiment. The obtained 2D ZQ spectrum is shown in Fig. 10(b-f). As a result, we have proved by direct observation of the splitting in the $2 \mathrm{D}$ spectrum that indeed $\Delta J_{\mathrm{HH}}^{\mathrm{Pa}}=\left|J_{\mathrm{H}^{\mathrm{c}}}^{\mathrm{Pa}}-J_{\mathrm{H}^{\mathrm{t}}}^{\mathrm{a}}\right|=2.3 \mathrm{~Hz}$, hence $\operatorname{sign}\left(J_{\mathrm{H}^{\mathrm{c}}}^{\mathrm{P}^{\mathrm{a}}}\right)=\operatorname{sign}\left(J_{\mathrm{H}^{\mathrm{t}}}^{\mathrm{P}^{\mathrm{a}}}\right)$. The projection of the $2 \mathrm{D}$ spectrum on the indirect dimension coincides with the spectrum shown in Fig. 6 for the $\mathrm{AXYZ}_{2}$ spin system: it is a doublet of triplets with resonance frequency $\delta v$ and splitting constants equal to 
$\Delta J_{\mathrm{HH}}^{\mathrm{P}}$ and $\Delta J_{\mathrm{HH}}^{\mathrm{Pe}}$. So we have shown that 2D ZQ spectroscopy can be applied not only to photo-PHIP complexes, but it is a general method that can be easily applied to any molecules of choice because the APSOC approach can be applied for weakly and strongly coupled spin systems equally efficiently. ${ }^{23,36}$

\section{Conclusions}

In summary, a comprehensive description of the spin dynamics of the photo-PHIP hyperpolarization technique is given. However, the description is not limited to photo-PHIP complexes and experiments, but can be applied to arbitrary spin systems and sequences that prepare a system in its pure singlet spin state. The theory is proved by experimental observation of the NMR spectra of $\mathrm{ZQ}_{\mathrm{X}}$ and $\mathrm{ZQ}_{\mathrm{Y}}$ coherences that were obtained from Fourier analysis of the spectral variation with the detection angle $\varphi$. The observation confirms experimentally the presence of two spectral components in photo-PHIP NMR spectra.

Modulation of ZQCs and $\rho_{\mathrm{S}}$ due to spin-spin interaction with hetero-nuclei that was incorrectly interpreted in earlier photo-PHIP work $^{8}$ was observed and explained. Analysis of oscillations and modulations of ZQCs allows us to formulate the first principles of ZQ spectroscopy. It provides one with information on the relative sign of $J$-coupling of two nuclei with a third one.

Exploitation of the S2M technique based on APSOC for direct observation of singlet spin order in photo-PHIP experiments is suggested. The APSOC method (1) simplifies the analysis of photo-PHIP spectra, because it converts anti-phase to in-phase spectra, (2) gives the same information on magnetic interactions as direct observation of ZQCs and (3) drastically improves SNR in case of low magnetic field homogeneity.

We suggest using the $2 \mathrm{D} Z \mathrm{ZQ}$ sequence based on the APSOC M2S block to obtain 2D ZQ spectra in a more convenient way than in the photo-PHIP experiment because it becomes unnecessary to bubble the sample with para-hydrogen and modify commercial NMR probes for irradiating the sample. Moreover, the sequence is applicable not only to photo-PHIP complexes but also to arbitrary spin systems that can be used to analyze polarization pathways in products of $\mathrm{pH}_{2}$ reactions of hydrogenation.

We are convinced that the methods developed and applied here will be helpful in future investigations of photo-PHIP and other para-hydrogen based hyperpolarization methods such as SABRE $^{13}$ and molecular tweezers. ${ }^{15}$

\section{Conflicts of interest}

There are no conflicts to declare.

\section{Acknowledgements}

The studies were supported by the Russian Science Foundation (project no. 15-13-20035). The authors acknowledge FASO Russia (project 0333-2016-0001) for financing the NMR facility.

\section{Notes and references}

1 K. H. Hausser and D. Stehlik, in Adv. Magn. Res., ed. J. S. Waugh, Academic, New York, 1968, vol. 3, pp. 79-139.

2 T. Maly, G. T. Debelouchina, V. S. Bajaj, K.-N. Hu, C.-G. Joo, M. L. Mak-Jurkauskas, J. R. Sirigiri, P. C. A. van der Wel, J. Herzfeld, R. J. Temkin and R. G. Griffin, J. Chem. Phys., 2008, 128, 052211.

3 W. Happer, Rev. Mod. Phys., 1972, 44, 169-249.

4 G. Navon, Y. Q. Song, T. Room, S. Appelt, R. E. Taylor and A. Pines, Science, 1996, 271, 1848-1851.

5 K. M. Salikhov, Y. N. Molin, R. Z. Sagdeev and A. L. Buchachenko, Spin polarization and magnetic effects in chemical reactions, Elsevier, Amsterdam, 1984.

6 C. R. Bowers and D. P. Weitekamp, Phys. Rev. Lett., 1986, 57, 2645-2648.

7 R. A. Green, R. W. Adams, S. B. Duckett, R. E. Mewis, D. C. Williamson and G. G. R. Green, Prog. Nucl. Magn. Reson. Spectrosc., 2012, 67, 1-48.

8 O. Torres, B. Procacci, M. E. Halse, R. W. Adams, D. Blazina, S. B. Duckett, B. Eguillor, R. A. Green, R. N. Perutz and D. C. Williamson, J. Am. Chem. Soc., 2014, 136, 10124-10131.

9 J. Natterer and J. Bargon, Prog. Nucl. Magn. Reson. Spectrosc., 1997, 31, 293-315.

10 K. V. Kovtunov, M. L. Truong, D. A. Barskiy, I. V. Koptyug, A. M. Coffey, K. W. Waddell and E. Y. Chekmenev, Chem. Eur. J., 2014, 20, 14629-14632.

11 C. R. Bowers and D. P. Weitekamp, J. Am. Chem. Soc., 1987, 109, 5541-5542.

12 E. A. Nasibulov, A. N. Pravdivtsev, A. V. Yurkovskaya, N. N. Lukzen, H.-M. Vieth and K. L. Ivanov, Z. Phys. Chem., 2013, 227, 929-953.

13 R. W. Adams, J. A. Aguilar, K. D. Atkinson, M. J. Cowley, P. I. P. Elliott, S. B. Duckett, G. G. R. Green, I. G. Khazal, J. López-Serrano and D. C. Williamson, Science, 2009, 323, 1708-1711.

14 F. Reineri, T. Boi and S. Aime, Nat. Commun., 2015, 6, 5858. 15 V. V. Zhivonitko, V. V. Telkki, K. Chernichenko, T. Repo, M. Leskela, V. Sumerin and I. V. Koptyug, J. Am. Chem. Soc., 2014, 136, 598-601.

16 T. Theis, G. X. Ortiz, A. W. J. Logan, K. E. Claytor, Y. Feng, W. P. Huhn, V. Blum, S. J. Malcolmson, E. Y. Chekmenev, Q. Wang and W. S. Warren, Sci. Adv., 2016, 2, e1501438.

17 M. H. Levitt, Annu. Rev. Phys. Chem., 2012, 63, 89-105.

18 R. Sarkar, P. Ahuja, P. R. Vasos, A. Bornet, O. Wagnieres and G. Bodenhausen, Prog. Nucl. Magn. Reson. Spectrosc., 2011, 59, 83-90.

19 M. E. Halse, B. Procacci, S.-L. Henshaw, R. N. Perutz and S. B. Duckett, J. Magn. Reson., 2017, 278, 25-38.

20 D. Blazina, S. B. Duckett, T. K. Halstead, C. M. Kozak, R. J. K. Taylor, M. S. Anwar, J. A. Jones and H. A. Carteret, Magn. Reson. Chem., 2005, 43, 200-208.

21 H. Samouei and V. V. Grushin, Organometallics, 2013, 32, 4440-4443.

22 R. Kaptein, in Biological Magnetic Resonance, ed. L. J. Berliner and J. Reuben, Springer US, Boston, MA, 1982, vol. 4, pp. 145-191, DOI: 10.1007/978-1-4615-6540-6_3. 
23 A. N. Pravdivtsev, A. S. Kiryutin, A. V. Yurkovskaya, H.-M. Vieth and K. L. Ivanov, J. Magn. Reson., 2016, 273, 56-64.

24 G. Pileio, M. Carravetta, E. Hughes and M. H. Levitt, J. Am. Chem. Soc., 2008, 130, 12582-12583.

25 S. J. DeVience, R. L. Walsworth and M. S. Rosen, Phys. Rev. Lett., 2013, 111, 173002.

26 J. Keller, Understanding NMR Spectroscopy, Willey, University of Cambridge, 1st edn, 2002.

27 M. H. Levitt, Spin dynamics: basics of nuclear magnetic resonance, Wiley, 2 edn, 2008.

28 O. W. Sørensen, G. W. Eich, M. H. Levitt, G. Bodenhausen and R. R. Ernst, Prog. Nucl. Magn. Reson. Spectrosc., 1983, 16, 163-192.

29 K. L. Ivanov, K. Miesel, H.-M. Vieth, A. V. Yurkovskaya and R. Z. Sagdeev, Z. Phys. Chem., 2003, 217, 1641-1659.

30 A. N. Pravdivtsev, Application for computation of NMR spectra, relaxation and polarization transfer in various experiments, accessed 01.06.2017, http:/www.tomo.nsc.ru/ en/nmr/iRelax.

31 S. Korchak, K. Ivanov, A. Yurkovskaya and H. M. Vieth, J. Chem. Phys., 2010, 133, 194502.

32 K. Ivanov, A. Yurkovskaya and H.-M. Vieth, J. Chem. Phys., 2008, 129, 234513.

33 A. S. Kiryutin, K. L. Ivanov, A. V. Yurkovskaya, H.-M. Vieth and N. N. Lukzen, Phys. Chem. Chem. Phys., 2013, 15, 14248-14255.

34 D. A. Barskiy, O. G. Salnikov, R. V. Shchepin, M. A. Feldman, A. M. Coffey, K. V. Kovtunov, I. V. Koptyug and E. Y. Chekmenev, J. Phys. Chem. C, 2016, 120, 29098-29106.

35 M. C. D. Tayler and M. H. Levitt, Phys. Chem. Chem. Phys., 2011, 13, 5556-5560.

36 A. S. Kiryutin, A. N. Pravdivtsev, A. V. Yurkovskaya, H.-M. Vieth and K. L. Ivanov, J. Phys. Chem. B, 2016, 120, 11978-11986. 